\title{
DERIVATIONS WITH INVERTIBLE VALUES ON A MULTILINEAR POLYNOMIAL
}

\author{
TSIU-KWEN LEE
}

(Communicated by Maurice Auslander)

\begin{abstract}
Let $R$ be a semiprime $K$-algebra with unity, $d$ a nonzero derivation of $R$, and $f\left(x_{1}, \ldots, x_{t}\right)$ a monic multilinear polynomial over $K$ such that $d\left(f\left(a_{1}, \ldots, a_{t}\right)\right) \neq 0$ for some $a_{1}, \ldots, a_{t} \in R$. It is shown that if for every $r_{1}, \ldots, r_{t}$ in $R$ either $d\left(f\left(r_{1}, \ldots, r_{t}\right)\right)=0$ or $d\left(f\left(r_{1}, \ldots, r_{t}\right)\right)$ is invertible in $R$, then $R$ is either a division ring $D$ or $M_{2}(D)$, the ring of $2 \times 2$ matrices over $D$, unless $f\left(x_{1}, \ldots, x_{t}\right)$ is a central polynomial for $R$.

Moreover, if $R=M_{2}(D)$, where $2 R \neq 0$ and $f\left(x_{1}, \ldots, x_{t}\right)$ is not a central polynomial for $D$, then $d$ is an inner derivation of $R$.
\end{abstract}

In [4] Bergen, Herstein, and Lanski proved that if $R$ is a ring with unity and $d \neq 0$ is a derivation of $R$ such that for every $x \in R, d(x)=0$ or $d(x)$ is invertible in $R$, then except for a special case which occurs when $2 R=0, R$ must be a division ring $D$ or $M_{2}(D)$, the ring of $2 \times 2$ matrices over a division ring $D$. In [5] Bergen and Carini gave a generalization of this result to the case of a Lie ideal. More precisely, for the semiprime case they proved: Let $R$ be a semiprime ring with $1, U$ a noncentral Lie ideal of $R$ such that $d(U) \neq 0$, and $d(u)=0$ or $d(u)$ is invertible for every $u \in U$. Then $R$ is either $D$ or $M_{2}(D)$ for some division ring $D$. Moreover, if $R=M_{2}(D)$, where $D$ is not commutative and $2 R \neq 0$, then $d$ must be inner.

Since by [9, Theorem 1.5] every noncentral Lie ideal of a simple ring $R$ must contain all commutators $x y-y x$ with $x, y \in R$ except if $R$ is of characteristic 2 and is 4-dimensional over its center, it is natural to examine what happens when the Lie ideal in Bergen and Carini's theorem is replaced by a multilinear polynomial.

Throughout this paper $R$ always denotes a semiprime $K$-algebra with unity where $K$ is a commutative ring with 1 . A polynomial $f\left(x_{1}, \ldots, x_{t}\right)$ in $K\left\{x_{1}, x_{2}, \ldots\right\}$, the free $K$-algebra with indeterminates $x_{i}$, is called monic if $f\left(x_{1}, \ldots, x_{t}\right)$ contains some monomial with coefficient 1 . In this paper we shall prove the following

Main Theorem. Let $R$ be a semiprime $K$-algebra with unity, $d$ a nonzero derivation of $R$, and $f\left(x_{1}, \ldots, x_{t}\right)$ a monic multilinear polynomial over $K$ such that $d\left(f\left(a_{1}, \ldots, a_{t}\right)\right) \neq 0$ for some $a_{i} \in R$. Suppose that for every

Received by the editors November 15, 1991 and, in revised form, April 7, 1992.

1991 Mathematics Subject Classification. Primary 16A72, 16A38.

Key words and phrases. Semiprime rings, derivations, central polynomials, differential identities. 
$r_{1}, \ldots, r_{t}$ in $R$ either $d\left(f\left(r_{1}, \ldots, r_{t}\right)\right)=0$ or $d\left(f\left(r_{1}, \ldots, r_{t}\right)\right)$ is invertible in $R$. Then $R$ is either a division ring $D$ or $M_{2}(D)$, the ring of $2 \times 2$ matrices over $D$, unless $f\left(x_{1}, \ldots, x_{t}\right)$ is a central polynomial for $R$. Moreover, if $R=M_{2}(D)$, where $2 R \neq 0$ and $f\left(x_{1}, \ldots, x_{t}\right)$ is not a central polynomial for $D$, then $d$ must be an inner derivation of $R$.

Given two elements $a, b \in R,[a, b]$ will denote the element $a b-b a$; also for two subsets $A, B$ of $R,[A, B]$ is then the additive subgroup of $R$ generated by all $[a, b]$ for $a \in A$ and $b \in B . Z(R)$ (or $Z$ in brief) stands for the center of $R$. We also recall that a polynomial $f\left(x_{1}, \ldots, x_{t}\right) \in$ $K\left\{x_{1}, x_{2}, \ldots\right\}$ is called a central polynomial for $R$ if $f\left(r_{1}, \ldots, r_{t}\right) \in Z(R)$ for all $r_{1}, \ldots, r_{t} \in R$. For any subset $S$ of $R$, denote by $l_{R}(S)$ the left annihilator of $S$ in $R$; that is, $l_{R}(S)=\{x \in R \mid x S=0\}$. We define $r_{R}(S)$ similarly.

We begin this paper with the following

Theorem 1. Let $R$ be a semiprime $K$-algebra with unity, $d$ a nonzero derivation of $R$, and $f\left(x_{1}, \ldots, x_{t}\right)$ a monic polynomial without constant term, not necessarily multilinear, over $K$ such that $d\left(f\left(a_{1}, \ldots, a_{t}\right)\right) \neq 0$ for some $a_{i} \in R$ and $f\left(x_{1}, \ldots, x_{t}\right)$ is not a central polynomial for $R$. Suppose that for every $r_{1}, \ldots, r_{t} \in R$ either $d\left(f\left(r_{1}, \ldots, r_{t}\right)\right)=0$ or $d\left(f\left(r_{1}, \ldots, r_{t}\right)\right)$ is invertible in $R$. Then $R$ is either

(I) a division ring $D$,

(II) $M_{2}(D)$, the ring of $2 \times 2$ matrices over a division ring $D$, or

(III) $M_{n}(\Delta)$ for some finite-dimensional central division algebra $\Delta$ and some positive integer $n$.

Moreover, if $f\left(b_{1}, \ldots, b_{t}\right)$ is an element in $R$ of rank $m \geq 1$ for some $b_{i} \in R$, then $R$ assumes (III) only if $n \leq 2 m$.

Proof. We first claim that $R$ is a simple ring with 1 . Indeed, let $I$ be a proper ideal of $R$ and $y_{1}, \ldots, y_{t} \in I^{2}$. Then it is clear that $d\left(f\left(y_{1}, \ldots, y_{t}\right)\right) \in$ $I$. Since either $d\left(f\left(y_{1}, \ldots, y_{t}\right)\right)=0$ or $d\left(f\left(y_{1}, \ldots, y_{t}\right)\right)$ is invertible, we have $d\left(f\left(y_{1}, \ldots, y_{t}\right)\right)=0$ for all $y_{i} \in I^{2}$. By [12, Theorem 4] we have $d\left(f\left(x_{1}, \ldots, x_{t}\right)\right) y=0$ for all $x_{i} \in R$ and all $y \in I^{2}$. But by hypothesis $d\left(f\left(a_{1}, \ldots, a_{t}\right)\right)$ is invertible; this implies $I^{2}=0$ and hence $I=0$ by the semiprimeness of $R$. Thus $R$ is a simple ring. We divide the proof into two cases.

Case 1. Assume that there exists a nonzero right ideal $\rho$ of $R$ such that $d\left(f\left(x_{1}, \ldots, x_{t}\right)\right)=0$ for all $x_{i} \in \rho$.

Denote by $g\left(x_{1}, \ldots, x_{m}\right)$ the multilinearization of $f\left(x_{1}, \ldots, x_{t}\right)$. Since $f \neq 0$ we have $g \neq 0$. By assumption $d\left(g\left(x_{1}, \ldots, x_{m}\right)\right)=0$ is an identity for $\rho$. Thus for any $u \in \rho$ we have

$$
\sum_{i=1}^{m} g\left(u x_{1}, \ldots, u d\left(x_{i}\right)+d(u) x_{i}, \ldots, u x_{m}\right)=0
$$

for all $x_{i} \in R$. If $d$ is an outer derivation of $R$, by Kharchenko's theorem [11] (1) is reduced to $\sum_{i=1}^{m} g\left(u x_{1}, \ldots, u y_{i}+d(u) x_{i}, \ldots, u x_{m}\right)=0$ for all $x_{i}, y_{i} \in R$. In particular, $g\left(u x_{1}, \ldots, u x_{m}\right)=0$ for all $x_{i} \in R$. If $\rho \subseteq Z(R)$, then $R$ is just a field and hence we are done. So we may assume $\rho \nsubseteq Z(R)$. Choose an element $u \in \rho \backslash Z(R)$. Then $g\left(u x_{1}, \ldots, u x_{m}\right)=0$ is a nontrivial generalized polynomial identity for $R$. Thus by Martindale's theorem [14] $R$ 
is a strongly primitive ring. Since $R$ is a simple ring with $1, R$ is a finitedimensional central simple algebra. That is, $R$ assumes the form (III). Thus we may assume that $d=a d(b)$, the inner derivation induced by some $b \in R$. That is, $d(x)=b x-x b$ for all $x \in R$. In this case, we have

$$
\left[b, g\left(x_{1}, \ldots, x_{m}\right)\right]=0 \text { for all } x_{i} \in \rho .
$$

Assume first that $(b-\alpha) \rho=0$ for some $\alpha \in Z(R)$. Choosing $u \in \rho \backslash Z(R)$ and using (2) we obtain that $g\left(u x_{1}, \ldots, u x_{m}\right)(b-\alpha)=0$ for all $x_{i} \in R$. Thus $R$ assumes the form (III) as before. So we assume that $(b-\alpha) \rho \neq 0$ for any $\alpha \in Z(R)$. Then there exists an element $u \in \rho$ such that $b u$ and $u$ are linearly independent over $Z(R)$. Now by (2) we yield that $b g\left(u x_{1}, \ldots, u x_{m}\right)-$ $g\left(u x_{1}, \ldots, u x_{m}\right) b=0$ is a nontrivial generalized polynomial identity for $R$. As before, $R$ assumes the form (III).

Case 2. Assume that $d(f(\rho)) \neq 0$ for all nonzero right ideals $\rho$ of $R$.

The proof of this case is essentially that of [4, Lemma 4]. For any nonzero right ideal $\rho$ of $R$ we have $0 \neq d(f(\rho)) \subseteq d(\rho) \rho+\rho \subseteq d(\rho)+\rho$ since $f$ has no constant term and $d(\rho)+\rho$ is a right ideal of $R$. But $0 \neq d(f(\rho))$ contains invertible values; this implies $d(\rho)+\rho=R$. Let $\rho_{1}, \rho_{2}$ be right ideals of $R$ such that $0 \neq \rho_{1} \subset \rho_{2}$. We want to prove that $\rho_{2}=R$. Indeed, this will imply that $R$ always assumes either (I) or (II). Note that $d\left(\rho_{1}\right)+\rho_{1}=R=d\left(\rho_{2}\right)+\rho_{2}$. Choose an element $t \in \rho_{2} \backslash \rho_{1}$. Write $t=a+d(b)$ for some $a, b \in \rho_{1}$. Then $d(b) \neq 0$ and $d(b)=t-a \in \rho_{2}$. Since $b R$ is a nonzero right ideal of $R$, we have $b R+d(b R)=R$. But $d(b R) \subseteq b d(R)+d(b) R \subseteq \rho_{2}$, thus we have $R=b R+d(b R) \subseteq \rho_{2}$, and hence $R=\rho_{2}$ as desired. This completes the first part of the theorem.

Finally, suppose that $R=M_{n}(\Delta)$ as given in (III) and that $\operatorname{rank}\left(f\left(b_{1}, \ldots, b_{t}\right)\right)$ $=m \geq 1$ for some $b_{i} \in R$. We want to prove $n \leq 2 m$. Assume on the contrary that $n>2 m$. Since $\operatorname{rank}\left(f\left(b_{1}, \ldots, b_{t}\right)\right)=m, f\left(b_{1}, \ldots, b_{t}\right)=g x$ for some $x \in R$ and some idempotent $g \in R$ with rank $m$. Thus $d\left(f\left(b_{1}, \ldots, b_{t}\right)\right)=$ $d(g x)=g d(g x)+d(g) g x$ and hence $\operatorname{rank}\left(d\left(f\left(b_{1}, \ldots, b_{t}\right)\right)\right) \leq 2 m$. So $d\left(f\left(b_{1}, \ldots, b_{t}\right)\right)=0$. Now consider the additive subgroup $A$ of $R$ generated by elements of rank $m$ assuming the form $f\left(u_{1}, \ldots, u_{t}\right)$ for some $u_{i} \in R$. Since $f\left(b_{1}, \ldots, b_{t}\right) \in A, A$ is a noncentral additive subgroup of $R$. Clearly, $A$ is invariant under special automorphisms in the sense of [7]. Thus by [7, Theorem 1] $A$ contains a noncentral Lie ideal of $R$, i.e., $A \supseteq[R, R]$. But $d(A)=0$, thus we get $d([R, R])=0$. Also, $\operatorname{dim}_{Z} R>4 m^{2}$, which implies $d=0$, a contradiction. So $n \leq 2 m$ as desired. This completes the proof.

To prove the Main Theorem we need some notation from [13]. Let $S$ be a ring with 1 and let $e_{i j}$ be the usual matrix units in the $n \times n$ matrix ring $M_{n}(S)$. Recall that for a sequence $u=\left(A_{1}, \ldots, A_{k}\right)$ in $M_{n}(S)$ the value of $u$ is defined to be the product $|u|=A_{1} A_{2} \cdots A_{k}$ and $u$ is nonvanishing if $|u| \neq 0$. For a permutation $\sigma$ of $\{1,2, \ldots, k\}$ we write $u^{\sigma}=\left(A_{\sigma(1)}, \ldots, A_{\sigma(k)}\right)$. We call $u$ simple if it has the form $u=\left(a_{1} e_{i_{1} j_{1}}, \ldots, a_{k} e_{i_{k} j_{k}}\right)$, where $a_{i} \in S$, $i=1, \ldots, k$. Finally, a simple sequence $u$ is called even if for some $\sigma$, $\left|u^{\sigma}\right|=b e_{i i} \neq 0$, and $o d d$ if for some $\sigma,\left|u^{\sigma}\right|=b e_{i j} \neq 0$, where $i \neq j$.

Before giving the proof of the Main Theorem we need the following result which is interesting in itself. The proof of the following lemma is implicit in [13, Lemma 2, proof of Lemma 3]. 
Lemma. Let $S$ be a $K$-algebra with 1 and let $R=M_{n}(S), n \geq 2$. Suppose that $h\left(x_{1}, \ldots, x_{t}\right)$ is a multilinear polynomial over $K$ such that $h(u)=0$ for all odd simple sequences $u$. Then $h\left(x_{1}, \ldots, x_{t}\right)$ is a central polynomial for $R$. Proof. Let $B_{1}, \ldots, B_{t} \in R$ be arbitrary. Since $h\left(x_{1}, \ldots, x_{t}\right)$ is multilinear, we have $h\left(B_{1}, \ldots, B_{t}\right)=\sum_{l=1}^{m} h\left(u^{(l)}\right)$, where the $u^{(l)}$ are even simple sequences because $h(u)=0$ for any odd simple sequence $u$. By [13, Lemma 2] each $h\left(u^{(l)}\right)$ assumes a diagonal form. Thus $h\left(B_{1}, \ldots, B_{t}\right)$ always assumes a diagonal form. Write

$$
h\left(B_{1}, \ldots, B_{t}\right)=\sum_{j=1}^{n} \beta_{j} e_{j j},
$$

where $\beta_{j} \in S$. For $1<k \leq n$ and any $\delta \in S$ we have that

$$
h\left(\left(1+\delta e_{1 k}\right) B_{1}\left(1+\delta e_{1 k}\right)^{-1}, \ldots,\left(1+\delta e_{1 k}\right) B_{t}\left(1+\delta e_{1 k}\right)^{-1}\right)
$$

still assumes a diagonal form. However,

$$
\begin{aligned}
h((1+ & \left.\left.\delta e_{1 k}\right) B_{1}\left(1+\delta e_{1 k}\right)^{-1}, \ldots,\left(1+\delta e_{1 k}\right) B_{t}\left(1+\delta e_{1 k}\right)^{-1}\right) \\
& =\left(1+\delta e_{1 k}\right) h\left(B_{1}, \ldots, B_{t}\right)\left(1+\delta e_{1 k}\right)^{-1} \\
& =\sum_{j=1}^{n} \beta_{j} e_{j j}+\left(\delta \beta_{k}-\beta_{1} \delta\right) e_{1 k}, \quad \text { since }\left(1+\delta e_{1 k}\right)^{-1}=1-\delta e_{1 k} .
\end{aligned}
$$

Thus $\delta \beta_{k}=\beta_{1} \delta$ for all $\delta \in S$. In particular, set $\delta=1$; then $\beta_{1}=\beta_{k}$. So $\delta \beta_{1}=\beta_{1} \delta$ for all $\delta \in S$, which implies $\beta_{1} \in Z(S)$. Now $h\left(B_{1}, \ldots, B_{t}\right)=$ $\beta_{1} \cdot \sum_{j=1}^{n} e_{j j} \in Z(R)$ as desired. This completes the proof.

Proof of Main Theorem. Assume that $f\left(x_{1}, \ldots, x_{t}\right)$ is not a central polynomial for $R$. By Theorem $1, R=M_{n}(\Delta)$ for some division ring $\Delta$, and to prove $n \leq 2$ it suffices to show that $\operatorname{rank}\left(f\left(b_{1}, \ldots, b_{t}\right)\right)=1$ for some $b_{i} \in R$. By the previous lemma there exists an odd simple sequence $u$ such that $f(u) \neq 0$. But by [13, Lemma 2] $f(u)=\mu e_{i j} \neq 0$ for some $\mu \in \Delta, i \neq j$; we get $\operatorname{rank}(f(u))=1$ as claimed.

Suppose next that $R=M_{2}(D)$, where $2 R \neq 0$ and $f\left(x_{1}, \ldots, x_{t}\right)$ is not a central polynomial for the division ring $D$. We want to prove that $d$ is inner. To do this we will refer to some arguments given in [4, Lemma 8; 5, Lemma 10]. Since $d$ is a derivation of $R, d$ has the form:

$$
d\left(\begin{array}{ll}
a & b \\
c & e
\end{array}\right)=\left(\begin{array}{cc}
\phi(a)-b \beta-\alpha c & \phi(b)+a \alpha+b \gamma-\alpha e \\
\phi(c)+\beta a-e \beta-\gamma c & \phi(e)+e \gamma-\gamma e+\beta b+c \alpha
\end{array}\right)
$$

for all $a, b, c, e \in D$, where $\alpha, \beta, \gamma \in D$ and $\phi$ is a derivation of $D$. Furthermore, by [4, Lemma 7] $d$ is inner on $M_{2}(D)$ if and only if $\phi$ is inner on $D$. Thus the aim is to prove that $\phi$ is inner on $D$. Suppose that $\alpha=0$. Then for $\beta_{1}, \ldots, \beta_{t} \in D$ we have

$$
\begin{aligned}
& d\left(f\left(\left(\begin{array}{cc}
\beta_{1} & 0 \\
0 & 0
\end{array}\right), \ldots,\left(\begin{array}{cc}
\beta_{t} & 0 \\
0 & 0
\end{array}\right)\right)\right) \\
& \quad=d\left(\begin{array}{cc}
f\left(\beta_{1}, \ldots, \beta_{t}\right) & 0 \\
0 & 0
\end{array}\right)=\left(\begin{array}{cc}
\phi\left(f\left(\beta_{1}, \ldots, \beta_{t}\right)\right) & 0 \\
\beta f\left(\beta_{1}, \ldots, \beta_{t}\right) & 0
\end{array}\right),
\end{aligned}
$$

which is zero or invertible. Thus $\phi\left(f\left(\beta_{1}, \ldots, \beta_{t}\right)\right)=0$ for all $\beta_{j} \in D$. Let $T$ denote the subdivision ring of $D$ generated by all elements $f\left(\beta_{1}, \ldots, \beta_{t}\right)$, 
where $\beta_{i} \in D$. Thus $\phi(T)=0$. Since $f$ is noncentral on $D, T$ is then a noncentral subdivision ring of $D$ invariant under all automorphisms. By a result of Brauer-Cartan-Hua [6], $T=D$ follows. Thus $\phi(D)=0$, implying that $\phi$ is inner. So we assume from now on that $\alpha \neq 0$. By (1) we have for $a \in D$ that

$$
d\left(\begin{array}{cc}
a & 0 \\
\alpha^{-1} \phi(a) & \alpha^{-1} a \alpha
\end{array}\right)=\left(\begin{array}{ll}
0 & 0 \\
u & v
\end{array}\right)
$$

where

$$
\begin{aligned}
& u=\phi\left(\alpha^{-1} \phi(a)\right)+\beta a-\alpha^{-1} a \alpha \beta-\gamma \alpha^{-1} \phi(a), \\
& v=\phi\left(\alpha^{-1} a \alpha\right)+\alpha^{-1} a \alpha \gamma-\gamma \alpha^{-1} a \alpha+\alpha^{-1} \phi(a) \alpha .
\end{aligned}
$$

Note that for $a, b \in D$ we have

$$
\left(\begin{array}{cc}
a & 0 \\
\alpha^{-1} \phi(a) & \alpha^{-1} a \alpha
\end{array}\right)\left(\begin{array}{cc}
b & 0 \\
\alpha^{-1} \phi(b) & \alpha^{-1} b \alpha
\end{array}\right)=\left(\begin{array}{cc}
a b & 0 \\
\alpha^{-1} \phi(a b) & \alpha^{-1} a b \alpha
\end{array}\right) .
$$

Thus for $\beta_{1}, \ldots, \beta_{t} \in D$ we have

$$
\begin{aligned}
& d\left(\begin{array}{cc}
f\left(\beta_{1}, \ldots, \beta_{t}\right) & 0 \\
\alpha^{-1} \phi\left(f\left(\beta_{1}, \ldots, \beta_{t}\right)\right) & \alpha^{-1} f\left(\beta_{1}, \ldots, \beta_{t}\right) \alpha
\end{array}\right) \\
& \quad=d\left(f\left(\left(\begin{array}{cc}
\beta_{1} & 0 \\
\alpha^{-1} \phi\left(\beta_{1}\right) & \alpha^{-1} \beta_{1} \alpha
\end{array}\right), \ldots,\left(\begin{array}{cc}
\beta_{t} & 0 \\
\alpha^{-1} \phi\left(\beta_{t}\right) & \alpha^{-1} \beta_{t} \alpha
\end{array}\right)\right)\right),
\end{aligned}
$$

which is either 0 or invertible. By (2) it must be zero. Now using the same calculations given in $[4$, Lemma 8$]$ we have

$$
\phi\left(f\left(\beta_{1}, \ldots, \beta_{t}\right)\right)=\frac{1}{2}\left[\phi(\alpha) \alpha^{-1}+\alpha \gamma \alpha^{-1}, f\left(\beta_{1}, \ldots, \beta_{t}\right)\right]
$$

for all $\beta_{1}, \ldots, \beta_{t} \in D$. Assume on the contrary that $\phi$ is outer on $D$. Since $f$ is multilinear, we have

$$
\sum_{j=1}^{t} f\left(\beta_{1}, \ldots, \phi\left(\beta_{j}\right), \ldots, \beta_{t}\right)=\frac{1}{2}\left[\phi(\alpha) \alpha^{-1}+\alpha \gamma \alpha^{-1}, f\left(\beta_{1}, \ldots, \beta_{t}\right)\right]
$$

for all $\beta_{i} \in D$. Applying Kharchenko's theorem [11] we have that

$$
\sum_{j=1}^{t} f\left(x_{1}, \ldots, y_{j}, \ldots, x_{t}\right)=\frac{1}{2}\left[\phi(\alpha) \alpha^{-1}+\alpha \gamma \alpha^{-1}, f\left(x_{1}, \ldots, x_{t}\right)\right]
$$

for all $x_{i}, y_{i} \in D$. In particular, taking $y_{1}=\cdots=y_{t}=0$ we get

$$
\frac{1}{2}\left[\phi(\alpha) \alpha^{-1}+\alpha \gamma \alpha^{-1}, f\left(x_{1}, \ldots, x_{t}\right)\right]=0 \text { for all } x_{i} \in D .
$$

Thus (4) is reduced to $\sum_{j=1}^{t} f\left(x_{1}, \ldots, y_{j}, \ldots, x_{t}\right)=0$ for all $x_{i}, y_{i} \in D$. So in particular $f\left(x_{1}, \ldots, x_{t}\right)=0$ for all $x_{i} \in D$, a contradiction. This completes the proof of the theorem.

With the Main Theorem in hand, the following question is naturally raised: Let $R$ be a semiprime $K$-algebra and $f\left(x_{1}, \ldots, x_{t}\right)$ a monic polynomial over $K$. Suppose that $d$ is a derivation of $R$ such that $d\left(f\left(x_{1}, \ldots, x_{t}\right)\right)=0$ for all $x_{i} \in R$. Then what can we say about the structure of $R$ ?

We conclude this paper with a precise description for the above question. 
Theorem 2. Let $R$ be a semiprime $K$-algebra with center $Z, Q$ the Martindale two-sided quotient ring of $R$, and $f\left(x_{1}, \ldots, x_{t}\right)$ a monic polynomial over $K$. Suppose that $d\left(f\left(x_{1}, \ldots, x_{t}\right)\right) \in Z$ for all $x_{1}, \ldots, x_{t} \in R$. Then there is a ring decomposition $Q=Q_{1} \oplus Q_{2} \oplus Q_{3}$ satisfying

(I) $d\left(Q_{1}\right)=0$,

(II) $Q_{2}$ satisfies $S_{4}$, the standard polynomial of degree 4 , and

(III) $f\left(x_{1}, \ldots, x_{t}\right)$ is a central polynomial for $Q_{3}$.

Proof. Denote by $C$ the extended centroid of $R$; then $Z(Q)=C$. It is well known that $d$ can be uniquely extended to $Q$. By [12, Theorem 3] $Q$ and $R$ satisfy the same differential identities. Thus we have $d\left(f\left(x_{1}, \ldots, x_{t}\right)\right) \in C$ for all $x_{i} \in Q$. Let $\mathscr{M}$ be any maximal ideal of $B$, the complete Boolean algebra of idempotents of $C$ [2]. Then $\mathscr{M} Q$ is a $d$-invariant prime ideal of $Q$. Let $\bar{d}$ denote the canonical derivation of $\bar{Q}=Q / \mathscr{M} Q$ induced by $d$. Note that $Z(\bar{Q})=(C+\mathscr{M} Q) / \mathscr{M} Q \cong C / \mathscr{M} C$. Thus $\bar{d}\left(f\left(x_{1}, \ldots, x_{t}\right)\right) \in(C+\mathscr{M} Q) / \mathscr{M} Q$ for all $x_{i} \in \bar{Q}$. It follows from [8, Theorem 3; Lemma 6; 10, Lemma 2] that either $f\left(x_{1}, \ldots, x_{t}\right)$ is a central polynomial for $\bar{Q}$, or $\bar{Q}$ satisfies $S_{4}$, or $\bar{d}=0$. Thus we have $d(Q) Q S_{4}\left(z_{1}, z_{2}, z_{3}, z_{4}\right) Q\left[f\left(x_{1}, \ldots, x_{t}\right), y\right] \subseteq \mathscr{M} Q$ for all $x_{i}, y, z_{i} \in Q$. But since $\bigcap\{\mathscr{M} Q \mid \mathscr{M}$ is any maximal ideal of $B\}=0$, we obtain

$$
d(Q) Q S_{4}\left(z_{1}, z_{2}, z_{3}, z_{4}\right) Q\left[f\left(x_{1}, \ldots, x_{t}\right), y\right]=0 \text { for all } x_{i}, y, z_{i} \in Q .
$$

By [2, Point 2] there exists an idempotent $h \in C$ such that $\{\alpha \in C \mid \operatorname{ad}(Q)=$ $0\}=h C$. Then $d(h Q)=h d(Q)=0$ and

$$
S_{4}\left(z_{1}, z_{2}, z_{3}, z_{4}\right)(1-h) Q\left[f\left(x_{1}, \ldots, x_{t}\right), y\right]=0
$$

for all $x_{i}, z_{i}, y \in(1-h) Q$. But $(1-h) Q$ is still an orthogonally complete ring; there exists an idempotent $g \in(1-h) C$ such that

$$
\left\{\beta \in(1-h) C \mid \beta S_{4}\left(z_{1}, z_{2}, z_{3}, z_{4}\right)=0 \text { for all } z_{i} \in(1-h) Q\right\}=g C .
$$

So $g Q$ satisfies $S_{4}$ and $(1-h)(1-g) Q$ satisfies $\left[f\left(x_{1}, \ldots, x_{t}\right), y\right]$. Now set $Q_{1}=h Q, Q_{2}=g Q$, and $Q_{3}=(1-h)(1-g) Q$. Then $Q=Q_{1} \oplus Q_{2} \oplus Q_{3}$ as desired. This completes the proof.

\section{REFERENCES}

1. K. I. Beidar, Rings with generalized identities. I, Vestnik Moskov. Univ. Ser. I Mat. Mekh. 32 (1977), 19-26; English transl. in Transl. Moscow Univ. Math. Bull. 32 (1977), 15-20.

2. _ Rings of quotients of semiprime rings, Vestnik Moskov. Univ. Ser. I Mat. Mekh. 33 (1978), 36-42; English transl. in Transl. Moscow Univ. Math. Bull. 33 (1978), 29-34.

3. J. Bergen, I. N. Herstein, and J. W. Kerr, Lie ideals and derivations of prime rings, J. Algebra 71 (1981), 259-267.

4. J. Bergen, I. N. Herstein, and C. Lanski, Derivations with invertible values, Canad. J. Math. 35 (1983), 300-310.

5. J. Bergen and L. Carini, Derivations with invertible values on a Lie ideal, Canad. Math. Bull. 31 (1988), 103-110.

6. R. Brauer, On a theorem of H. Cartan, Bull. Amer. Math. Soc. 55 (1949), 619-620.

7. C. L. Chuang, On invariant additive subgroups, Israel J. Math. 57 (1987), 116-128.

8. __ The additive subgroup generated by a polynomial, Israel J. Math. 59 (1987), 98-106.

9. I. N. Herstein, Topics in ring theory, Univ. of Chicago Press, Chicago, 1969. 
10. W. F. Ke, On derivations of prime rings of characteristic 2, Chinese J. Math. 13 (1985), 273-290.

11. V. K. Kharchenko, Differential identities of prime rings, Algebra i Logika 17 (1978), 220238.

12. T. K. Lee, Semiprime rings with differential identities, Bull. Inst. Math. Acad. Sinica 20 (1992), 39-50.

13. U. Leron, Nil and power-central polynomials in rings, Trans. Amer. Math. Soc. 202 (1975), 97-103.

14. W. S. Martindale III, Prime rings satisfying a generalized polynomial identity, J. Algebra 12 (1969), 576-584.

Department of Mathematics, National Taiwan University, Taipei, Taiwan 10764, REPUBLIC OF CHINA 